\title{
Sharp Bounds for the General Sum-Connectivity Indices of Transformation Graphs
}

\author{
Haiying Wang, ${ }^{1}$ Jia-Bao Liu, ${ }^{2}$ Shaohui Wang, ${ }^{3}$ Wei Gao, ${ }^{4}$ Shehnaz Akhter, ${ }^{5}$ \\ Muhammad Imran, ${ }^{5,6}$ and Mohammad R. Farahani ${ }^{7}$ \\ ${ }^{1}$ School of Science, China University of Geosciences (Beijing), Beijing 100083, China \\ ${ }^{2}$ School of Mathematics and Physics, Anhui Jianzhu University, Hefei 230601, China \\ ${ }^{3}$ Department of Mathematics, Savannah State University, Savannah, GA 31404, USA \\ ${ }^{4}$ School of Information and Technology, Yunnan Normal University, Kunming 650500, China \\ ${ }^{5}$ Department of Mathematics, School of Natural Sciences (SNS), National University of Sciences and Technology (NUST), \\ Sector H-12, Islamabad, Pakistan \\ ${ }^{6}$ Department of Mathematical Sciences, College of Science, United Arab Emirates University, P.O. Box 15551, Al Ain, UAE \\ ${ }^{7}$ Department of Applied Mathematics, Iran University of Science and Technology (IUST), Narmak, Tehran 16844, Iran
}

Correspondence should be addressed to Haiying Wang; whycht@126.com

Received 12 July 2017; Revised 23 October 2017; Accepted 9 November 2017; Published 3 December 2017

Academic Editor: Chris Goodrich

Copyright (C) 2017 Haiying Wang et al. This is an open access article distributed under the Creative Commons Attribution License, which permits unrestricted use, distribution, and reproduction in any medium, provided the original work is properly cited.

Given a graph $G$, the general sum-connectivity index is defined as $\chi_{\alpha}(G)=\sum_{u v \in E(G)}\left(d_{G}(u)+d_{G}(v)\right)^{\alpha}$, where $d_{G}(u)\left(\right.$ or $\left.d_{G}(v)\right)$ denotes the degree of vertex $u$ (or $v$ ) in the graph $G$ and $\alpha$ is a real number. In this paper, we obtain the sharp bounds for general sum-connectivity indices of several graph transformations, including the semitotal-point graph, semitotal-line graph, total graph, and eight distinct transformation graphs $G^{u v w}$, where $u, v, w \in\{+,-\}$.

\section{Introduction}

In this paper, we consider simple, undirected, and connected graphs. Let $G$ be the graph with vertex set $V(G)$ and edge set $E(G)$. The order and size of $G$ are denoted by $n$ and $e$, respectively. For a vertex $a \in V(G), d_{G}(a)$ denotes the degree of $a$. Two vertices in $G$ are adjacent if and only if they are end vertices of an edge, and each of the two vertices is called incident to the edge. Besides, two edges are adjacent to each other if and only if they share a common vertex. The minimum and maximum degrees of graph $G$ are denoted by $\delta(G)$ and $\Delta(G)$, respectively. We will use the notations $P_{n}, C_{n}$, and $K_{n}$ for a path, cycle, and complete graph of order $n$ [1], respectively.

The complement of $G$, denoted by $\bar{G}$, is the graph with $V(\bar{G})=V(G)$ and two vertices in $\bar{G}$ are adjacent if and only if they are not adjacent in $G$. Thus, the size of $\bar{G}$ is $\left(\begin{array}{l}n \\ 2\end{array}\right)-e$ and if $a \in V(\bar{G})$ then $d_{\bar{G}}(a)=n-1-d_{G}(a)$.
A topological index is a numeric quantity associated with a graph which characterizes the topology of graph. A topological index $\operatorname{Top}(G)$ of a graph $G$ is equal to the topological index $\operatorname{Top}(H)$ of $H$, if and only if two graphs $G$ and $H$ are isomorphic. The idea of topological index appears from work done by Wiener in 1947, this index is called Wiener index. The first and second Zagreb indices have been introduced by Gutman and Trinajestić [2]. These indices are defined on the ground of vertex degrees as follows:

$$
\begin{aligned}
& M_{1}(G)=\sum_{a \in V(G)}\left(d_{G}(a)\right)^{2}, \\
& M_{2}(G)=\sum_{a b \in E(G)} d_{G}(a) d_{G}(b) .
\end{aligned}
$$

The Randić connectivity index was defined in 1975 by Randić [3]. It has been extended to the general Randić connectivity index. The general Randić connectivity index (general 
product-connectivity index) was defined by Bollobás and Erdős [4] as follows:

$$
R_{\alpha}(G)=\sum_{a b \in E(G)}\left(d_{G}(a) d_{G}(b)\right)^{\alpha},
$$

where $\alpha$ is a real number. Then $R_{-1 / 2}$ is the classical Randic connectivity index. The sum-connectivity index was proposed in [5]. This concept was extended to the general sumconnectivity index in [6], which is defined as

$$
\chi_{\alpha}(G)=\sum_{a b \in E(G)}\left(d_{G}(a)+d_{G}(b)\right)^{\alpha}
$$

where $\alpha$ is a real number. Then $\chi_{-1 / 2}(G)$ is the classical sum-connectivity index. The sum-connectivity index and the product-connectivity index correlate well with the $\pi$-electron energy of benzenoid hydrocarbons [7].

The total graph $T(G)$ of the graph $G$ is a graph whose vertex set is the union of $V(G)$ and $E(G)$ such that $a b \in$ $E(T(G))$ if and only if $a$ and $b$ are either adjacent or incident in $G$ [8]. Let $u, v$, and $w$ be the variables having values + or -. The transformation graph $G^{u v w}$ is a graph whose vertex set is the union of $V(G)$ and $E(G)$, and $a b \in E\left(G^{u v w}\right)$ if and only if

(1) $a, b \in V(G)$; then $u=+$ or $u=-$ if $a$ and $b$ are adjacent or nonadjacent in $G$, respectively;

(2) $a, b \in E(G)$; then $v=+$ or $v=-$ if $a$ and $b$ are adjacent or nonadjacent in $G$, respectively;

(3) $a \in V(G)$ and $b \in E(G)$; then $w=+$ or $w=-$ if $a$ and $b$ are incident or nonincident in $G$, respectively.

There are eight different transformations of the given graph $G$. For instance, $G^{+++}$is the total graph $T(G)$ of $G$ with number of vertices $n+e$ and number of edges $(1 / 2) M_{1}(G)+$ $2 e$, and $G^{---}$is the complement of total graph $G^{+++}$. For other transformations of graph, $G^{++-}, G^{+-+}$, and $G^{+--}$are the complements of $G^{--+}, G^{-+-}$, and $G^{-++}$, respectively.

The concepts of semitotal-point graph and semitotal-line graph are introduced by Sampathkumar and Chikkodimath [9]. The semitotal-point graph $T_{1}(G)$ is a graph whose vertex set is the union of $V(G)$ and $E(G)$, and $a b \in E\left(T_{1}(G)\right)$ if and only if (i) $a$ and $b$ are adjacent vertices in $G$ or (ii) one is a vertex of $G$ and the other is an edge of $G$ incident to it. Thus, semitotal-point graph has $n+e$ number of vertices and $3 e$ number of edges.

The semitotal-line graph $T_{2}(G)$ is a graph whose vertex set is the union of $V(G)$ and $E(G)$, and $a b \in E\left(T_{2}(G)\right)$ if and only if (i) $a$ and $b$ are adjacent edges in $G$ and (ii) one is a vertex of $G$ and the other is an edge of $G$ incident to it. Thus, semitotalline graph has $n+e$ number of vertices and $(1 / 2) M_{1}(G)+e$ number of edges.

Eventually, many properties of these transformation graphs can be determined. For example, the Zagreb indices of transformation graphs and total transformation graphs were calculated by Basavanagoud and Patil [10] and Hosamani and Gutman [11], respectively. Wu and Meng [12] investigated the basic properties (connectedness, graph equations and iteration, and diameter) of total transformation. $\mathrm{Xu}$ and $\mathrm{Wu}$ [13] determined the connectivity, the Hamiltonian, and the independence number of $G^{-+-}$. Yi and $\mathrm{Wu}$ [14] determined the connectivity, the Hamiltonian, and the independence number of $G^{++-}$.

In this paper, we obtain lower and upper bounds for the general sum-connectivity indices of the above-defined transformation graphs.

\section{Main Results}

In this section, we discuss the lower and upper bounds for the general sum-connectivity indices of transformation graphs defined in Section 1.

Theorem 1. For $\alpha<0$, we have $\gamma_{1} \leq \chi_{\alpha}\left(T_{1}(G)\right) \leq \gamma_{2}$, where

$$
\begin{aligned}
& \gamma_{1}=2^{\alpha} \chi_{\alpha}(G)+2^{\alpha+1} e(\Delta(G)+1)^{\alpha}, \\
& \gamma_{2}=2^{\alpha} \chi_{\alpha}(G)+2^{\alpha+1} e(\delta(G)+1)^{\alpha} ;
\end{aligned}
$$

the equalities hold if and only if $G$ is a regular graph.

Proof. Since $T_{1}(G)$ has $n+e$ vertices and $3 e$ edges, it holds that

$$
\begin{gathered}
\chi_{\alpha}\left(T_{1}(G)\right)=\sum_{a b \in E\left(T_{1}(G)\right)}\left(d_{T_{1}(G)}(a)+d_{T_{1}(G)}(b)\right)^{\alpha} \\
=\sum_{\substack{a b \in E\left(T_{1}(G)\right), a, b \in V(G)}}\left(d_{T_{1}(G)}(a)+d_{T_{1}(G)}(b)\right)^{\alpha} \\
\quad+\sum_{\substack{a b \in E\left(T_{1}(G)\right), a \in V(G), b \in E(G)}}\left(d_{T_{1}(G)}(a)+d_{T_{1}(G)}(b)\right)^{\alpha} .
\end{gathered}
$$

Note that if $a \in V(G)$ then $d_{T_{1}(G)}(a)=2 d_{G}(a)$ and if $a \in$ $E(G)$ then $d_{T_{1}(G)}(a)=2$. It is clear that $\delta(G) \leq d_{G}(a)$ and $\Delta(G) \geq d_{G}(a)$. And these equalities hold if and only if $G$ is a regular graph. Therefore,

$$
\begin{aligned}
\chi_{\alpha}\left(T_{1}(G)\right)= & 2^{\alpha} \sum_{\substack{a b \in E\left(T_{1}(G)\right), a, b \in V(G)}}\left(d_{G}(a)+d_{G}(b)\right)^{\alpha} \\
& +2^{\alpha} \sum_{\substack{a b \in E\left(T_{1}(G)\right), a \in V(G), b \in E(G)}}\left(d_{G}(a)+1\right)^{\alpha} \\
\geq & 2^{\alpha} \chi_{\alpha}(G)+2^{\alpha+1} e(\Delta(G)+1)^{\alpha} .
\end{aligned}
$$

Similarly, we can compute

$$
\chi_{\alpha}\left(T_{1}(G)\right) \leq 2^{\alpha} \chi_{\alpha}(G)+2^{\alpha+1} e(\delta(G)+1)^{\alpha} .
$$

The two equalities in (6) and (7) obviously hold if and only if $G$ and $H$ are regular, respectively.

Example 2. By Theorem 1, the general sum-connectivity indices of some semitotal-point graphs are given below:

(1) $n\left(8^{\alpha}+2 \times 6^{\alpha}\right)-3 \times 8^{\alpha} \leq \chi_{\alpha}\left(T_{1}\left(P_{n}\right)\right) \leq 2^{2 \alpha} n\left(2^{\alpha}+2\right)+$ $2 \times 6^{\alpha}-3 \times 8^{\alpha}-2^{2 \alpha+1}$. 
(2) $\chi_{\alpha}\left(T_{1}\left(C_{n}\right)\right)=2^{\alpha} n\left(4^{\alpha}+2 \times 3^{\alpha}\right)$.

(3) $\chi_{\alpha}\left(T_{1}\left(K_{n}\right)\right)=2^{\alpha} n(n-1)\left[2^{\alpha-1}(n-1)^{\alpha}+n^{\alpha}\right]$.

Theorem 3. If $\alpha<0$ then $\gamma_{1} \leq \chi_{\alpha}\left(T_{2}(G)\right) \leq \gamma_{2}$, where

$$
\begin{aligned}
& \gamma_{1}=2^{2 \alpha-1} M_{1}(G) \Delta^{\alpha}(G)+e \Delta^{\alpha}(G)\left[2 \times 3^{\alpha}-4^{\alpha}\right], \\
& \gamma_{2}=2^{2 \alpha-1} M_{1}(G) \delta^{\alpha}(G)+e \delta^{\alpha}(G)\left[2 \times 3^{\alpha}-4^{\alpha}\right] ;
\end{aligned}
$$

the equalities hold if and only if $G$ is a regular graph.

Proof. Since $\left|V\left(T_{2}(G)\right)\right|=n+e$ and $\left|E\left(T_{2}(G)\right)\right|=(1 /$ 2) $M_{1}(G)+e$, we have

$$
\begin{gathered}
\chi_{\alpha}\left(T_{2}(G)\right)=\sum_{a b \in E\left(T_{2}(G)\right)}\left(d_{T_{2}(G)}(a)+d_{T_{2}(G)}(b)\right)^{\alpha} \\
=\sum_{\substack{a b \in E\left(T_{2}(G)\right), a, b \in E(G)}}\left(d_{T_{2}(G)}(a)+d_{T_{2}(G)}(b)\right)^{\alpha} \\
+\sum_{\substack{a b \in E\left(T_{2}(G)\right), a \in V(G), b \in E(G)}}\left(d_{T_{2}(G)}(a)+d_{T_{2}(G)}(b)\right)^{\alpha} .
\end{gathered}
$$

Note that if $a \in E(G)$ then $d_{T_{2}(G)}(a)=d_{G}\left(w_{i}\right)+d_{G}\left(w_{j}\right)$ and if $a \in V(G)$ then $d_{T_{2}(G)}(a)=d_{G}(a)$. Therefore, we have

$$
\begin{gathered}
\chi_{\alpha}\left(T_{2}(G)\right)=\sum_{\substack{w_{i} w_{j} \in E(G), w_{j} w_{k} \in E(G), w_{i} \neq w_{k}}}\left[\left(d_{G}\left(w_{i}\right)+d_{G}\left(w_{j}\right)\right)\right. \\
\left.+\left(d_{G}\left(w_{j}\right)+d_{G}\left(w_{k}\right)\right)\right]^{\alpha}+\sum_{\substack{a b \in E\left(T_{2}(G)\right), a \in V(G), b=a x \in(G), x \in V(G)}}\left[d_{G}(a)\right. \\
\left.+\left(d_{G}(a)+d_{G}(x)\right)\right]^{\alpha}=\sum_{\substack{w_{i} w_{j} \in E(G), w_{j} w_{k} \in E(G), w_{i} \neq w_{k}}}\left[d_{G}\left(w_{i}\right)\right. \\
\left.+2 d_{G}\left(w_{j}\right)+d_{G}\left(w_{k}\right)\right]^{\alpha}+\sum_{\substack{a \in V(G), b=a x \in E(G), x \in V(G)}}\left[d_{G}(a)\right. \\
\left.+\left(d_{G}(a)+d_{G}(x)\right)\right]^{\alpha} .
\end{gathered}
$$

Since $d_{G}(a) \geq \delta(G)$ and $d_{G}(a) \leq \Delta(G)$, each equality holds if and only if $G$ is a regular graph.
After simplification we get

$$
\begin{aligned}
& \chi_{\alpha}\left(T_{2}(G)\right) \geq[4 \Delta(G)]^{\alpha} \sum_{\substack{w_{i} w_{j} \in E(G), w_{j} w_{k} \in\left(G(G), w_{i} \neq w_{k}\right.}} 1 \\
&+[3 \Delta(G)]^{\alpha} \sum_{\substack{a b \in E\left(T_{2}(G)\right), a \in V(G), b=a x \in E(G), x \in V(G)}} 1 \\
&=[4 \Delta(G)]^{\alpha} \cdot\left[E\left(T_{2}(G)\right)-2 e\right] \\
&+[3 \Delta(G)]^{\alpha} \cdot(2 e) \\
&= 2^{2 \alpha-1} M_{1}(G) \Delta^{\alpha}(G) \\
&+e \Delta^{\alpha}(G)\left[2 \times 3^{\alpha}-4^{\alpha}\right] .
\end{aligned}
$$

Similarly, we can calculate

$$
\begin{aligned}
\chi_{\alpha}\left(T_{2}(G)\right) \leq & 2^{2 \alpha-1} M_{1}(G) \delta^{\alpha}(G) \\
& +e \delta^{\alpha}(G)\left[2 \times 3^{\alpha}-4^{\alpha}\right] .
\end{aligned}
$$

Obviously the equalities in (11) and (12) hold if and only if $G$ is a regular graph.

Example 4. By Theorem 3, the general sum-connectivity indices of some semitotal-line graphs are given below:

$$
\begin{aligned}
& \text { (1) } 2^{\alpha} n\left(4^{\alpha}+2 \times 3^{\alpha}\right)-2^{\alpha+1}\left(4^{\alpha}-3^{\alpha}\right) \leq \chi_{\alpha}\left(T_{2}\left(P_{n}\right)\right) \leq n\left(4^{\alpha}+\right. \\
& \left.2 \times 3^{\alpha}\right)-2\left(4^{\alpha}+2 \times 3^{\alpha}\right) . \\
& \text { (2) } \chi_{\alpha}\left(T_{2}\left(C_{n}\right)\right)=2^{\alpha} n\left(4^{\alpha}+2 \times 3^{\alpha}\right) . \\
& \text { (3) } \chi_{\alpha}\left(T_{2}\left(K_{n}\right)\right)=n^{\alpha}(n-1)^{\alpha+1}\left[2^{2 \alpha-1} n+2^{2 \alpha}+3^{\alpha}\right] .
\end{aligned}
$$

Theorem 5. Let $\alpha<0$. Then $\gamma_{1} \leq \chi_{\alpha}(T(G)) \leq \gamma_{2}$, where

$$
\begin{aligned}
& \gamma_{1}=2^{\alpha} \chi_{\alpha}(G)+2^{2 \alpha-1} M_{1}(G) \Delta^{\alpha}(G)+4^{\alpha} e \Delta^{\alpha}(G), \\
& \gamma_{2}=2^{\alpha} \chi_{\alpha}(G)+2^{2 \alpha-1} M_{1}(G) \delta^{\alpha}(G)+4^{\alpha} e \delta^{\alpha}(G) ;
\end{aligned}
$$

the equalities hold if and only if $G$ is a regular graph.

Proof. Since $|V(T(G))|=n+e$ and $|E(T(G))|=(1 / 2) M_{1}(G)+$ $2 e$, we have

$$
\begin{aligned}
\chi_{\alpha}(T(G))= & \sum_{a b \in E(T(G))}\left(d_{T(G)}(a)+d_{T(G)}(b)\right)^{\alpha} \\
= & \sum_{\substack{a b \in E(T(G)), a, b \in V(G)}}\left(d_{T(G)}(a)+d_{T(G)}(b)\right)^{\alpha} \\
& +\sum_{\substack{a b \in E(T(G)), a, b \in E(G)}}\left(d_{T(G)}(a)+d_{T(G)}(b)\right)^{\alpha} \\
& +\sum_{\substack{a \in \in E(T(G)), a \in V(G), b \in E(G)}}\left(d_{T(G)}(a)+d_{T(G)}(b)\right)^{\alpha} .
\end{aligned}
$$


Note that $d_{T(G)}(a)=2 d_{G}(a)$ for $a \in V(G)$ and $d_{T(G)}(a)=$ $d_{G}\left(w_{i}\right)+d_{G}\left(w_{j}\right)$ for $a \in E(G)$. So

$$
\begin{aligned}
& \chi_{\alpha}(T(G))=2^{\alpha} \sum_{\substack{a b \in E(G), a, b \in V(G)}}\left(d_{G}(a)+d_{G}(b)\right)^{\alpha} \\
& +\sum_{\substack{a=w_{i} w_{j} \in E(G), b=w_{j} w_{k} \in E(G), w_{i} \neq w_{k}}}\left[\left(d_{G}\left(w_{i}\right)+d_{G}\left(w_{j}\right)\right)\right. \\
& \left.+\left(d_{G}\left(w_{j}\right)+d_{G}\left(w_{k}\right)\right)\right]^{\alpha}+\sum_{\substack{b=a x \in E(G), a \in V(G), x \in V(G)}}\left[2 d_{G}(a)\right. \\
& \left.+\left(d_{G}(a)+d_{G}(x)\right)\right]^{\alpha}=2^{\alpha} \cdot \chi_{\alpha}(G) \\
& +\sum_{\substack{a=w_{i} w_{j} \in E(G), b=w_{j} w_{k} \in E(G), w_{i} \neq w_{k}}}\left[d_{G}\left(w_{i}\right)+2 d_{G}\left(w_{j}\right)+d_{G}\left(w_{k}\right)\right]^{\alpha} \\
& +\sum_{\substack{b=a x \in E(G), a \in V(G), x \in V(G)}}\left[2 d_{G}(a)+\left(d_{G}(a)+d_{G}(x)\right)\right]^{\alpha} .
\end{aligned}
$$

Note that $d_{G}(a) \leq \Delta(G)$ and $d_{G}(a) \geq \delta(G)$. The equalities hold if and only if $G$ is a regular graph.

After simplification, we get

$$
\begin{aligned}
\chi_{\alpha}(T(G)) \geq & 2^{\alpha} \chi_{\alpha}(G)+[4 \Delta(G)]^{\alpha}\left[\frac{1}{2} M_{1}(G)-e\right] \\
& +[4 \Delta(G)]^{\alpha}[2 e] \\
= & 2^{\alpha} \chi_{\alpha}(G)+2^{2 \alpha-1} \Delta^{\alpha}(G) M_{1}(G) \\
& +2^{2 \alpha} e \Delta^{\alpha}(G) .
\end{aligned}
$$

Similarly, we can compute

$$
\begin{aligned}
\chi_{\alpha}(T(G)) \leq & 2^{\alpha} \chi_{\alpha}(G)+2^{2 \alpha-1} \delta^{\alpha}(G) M_{1}(G) \\
& +2^{2 \alpha} e \delta^{\alpha}(G) .
\end{aligned}
$$

Since $\chi_{\alpha}(G) \geq 2^{\alpha} e \Delta^{\alpha}(G)$, we can also write the results above as

$$
\begin{gathered}
4^{\alpha} \delta^{\alpha}(G)\left[\frac{1}{2} M_{1}(G)+2 e\right] \leq \chi_{\alpha}(T(G)) \\
\leq 4^{\alpha} \Delta^{\alpha}(G)\left[\frac{1}{2} M_{1}(G)+2 e\right] .
\end{gathered}
$$

Thus, if $G$ is a regular graph, then we obtain the equality in (16), (17), and (18).

Example 6. By Theorem 5, the general sum-connectivity indices of some total graphs are given below:

(1) $2^{3 \alpha+2} n+2 \times 6^{\alpha}-7 \times 8^{\alpha} \leq \chi_{\alpha}\left(T\left(P_{n}\right)\right) \leq 4^{\alpha} n\left(2^{\alpha}+1\right)+$ $2^{\alpha}\left(2 \times 3^{\alpha}-3 \times 4^{\alpha}+-2^{\alpha+2}\right)$.
(2) $\chi_{\alpha}\left(T\left(C_{n}\right)\right)=4 \times 8^{\alpha} n$.
(3) $\chi_{\alpha}\left(T\left(K_{n}\right)\right)=2^{2 \alpha-1} n(n-1)^{\alpha+1}(n+1)$.

Theorem 7. Let $\alpha<0$. Then $\gamma_{1} \leq \chi_{\alpha}\left(G^{---}\right) \leq \gamma_{2}$, where

$$
\begin{aligned}
& \gamma_{1}=2^{\alpha}\left[\frac{1}{2} M_{1}(G)+2 e\right]\left(e+n-1-2 \Delta^{\alpha}(G)\right)^{\alpha}, \\
& \gamma_{2}=2^{\alpha}\left[\frac{1}{2} M_{1}(G)+2 e\right]\left(e+n-1-2 \delta^{\alpha}(G)\right)^{\alpha}
\end{aligned}
$$

the equalities hold if and only if $G$ is a regular graph.

Proof. For a given graph $G$, since $G^{---} \cong \overline{G^{+++}}$and $G^{+++}=$ $T(G)$, then $\left|V\left(G^{---}\right)\right|=n+e,\left|E\left(G^{---}\right)\right|=\left(\begin{array}{c}e+n \\ 2\end{array}\right)-$ $(1 / 2) M_{1}(G)-2 e$, and $2 \Delta(\bar{G})=e+n-1-2 \Delta(G)$. Using these values, we can compute the required results.

Theorem 8. Let $\alpha<0$. Then $\gamma_{1} \leq \chi_{\alpha}\left(G^{++-}\right) \leq \gamma_{2}$, where

$$
\begin{aligned}
\gamma_{1}= & 2^{\alpha} e^{\alpha+1}+2^{\alpha}\left[\frac{1}{2} M_{1}(G)-e\right][n-4+2 \Delta(G)]^{\alpha} \\
& +\left[\left(\begin{array}{c}
e+n \\
2
\end{array}\right)-\left(\begin{array}{l}
n \\
2
\end{array}\right)-\left(\begin{array}{l}
e \\
2
\end{array}\right)-2 e\right] \\
& \cdot[e+n-4+2 \Delta(G)]^{\alpha}, \\
\gamma_{2}= & 2^{\alpha} e^{\alpha+1}+2^{\alpha}\left[\frac{1}{2} M_{1}(G)-e\right][n-4+2 \delta(G)]^{\alpha} \\
& +\left[\left(\begin{array}{c}
e+n \\
2
\end{array}\right)-\left(\begin{array}{l}
n \\
2
\end{array}\right)-\left(\begin{array}{l}
e \\
2
\end{array}\right)-2 e\right] \\
& \cdot[e+n-4+2 \delta(G)]^{\alpha} ;
\end{aligned}
$$

the equalities hold if and only if $G$ is a regular graph.

Proof. Since $\left|V\left(G^{++-}\right)\right|=n+e$ and $\left|E\left(G^{++-}\right)\right|=\left(\begin{array}{c}e+n \\ 2\end{array}\right)-\left(\begin{array}{c}n \\ 2\end{array}\right)-$ $\left(\begin{array}{l}e \\ 2\end{array}\right)+(1 / 2) M_{1}(G)-2 m$,

$$
\begin{aligned}
\chi_{\alpha}\left(G^{++-}\right)= & \sum_{a b \in E\left(G^{++}\right)}\left(d_{G^{++-}}(a)+d_{G^{++-}}(b)\right)^{\alpha} \\
= & \sum_{\substack{a b \in E\left(G^{++-}\right), a, b \in V(G)}}\left(d_{G^{++-}}(a)+d_{G^{++}}(b)\right)^{\alpha} \\
& +\sum_{\substack{a b \in E\left(G^{++-}\right), a, b \in E(G)}}\left(d_{G^{++-}}(a)+d_{G^{++-}}(b)\right)^{\alpha} \\
& +\sum_{\substack{a b \in E\left(G^{++-}\right), a \in V(G), b \in E(G)}}\left(d_{G^{++-}}(a)+d_{G^{++-}}(b)\right)^{\alpha} .
\end{aligned}
$$


Note that if $a \in V(G)$ then $d_{G^{++-}}(a)=e$ and if $a \in E(G)$ then $d_{G^{++}}(a)=d_{G}\left(w_{i}\right)+d_{G}\left(w_{j}\right)+n-4$

$$
\begin{aligned}
& \chi_{\alpha}\left(G^{++-}\right)=\sum_{\substack{a b \in E(G) \\
a, b \in V(G)}}[2 e]^{\alpha} \\
& +\sum_{\substack{a=w_{i} w_{j} \in E(G), b=w_{j} w_{k} \in E(G), w_{i} \neq w_{k}}}\left[\left(d_{G}\left(w_{i}\right)+d_{G}\left(w_{j}\right)+n-4\right)\right. \\
& \left.+\left(d_{G}\left(w_{j}\right)+d_{G}\left(w_{k}\right)+n-4\right)\right]^{\alpha}+\sum_{\substack{b=x y \in E(G), a \in V(G), a \notin\{x, y\}}}[e \\
& \left.+\left(d_{G}(x)+d_{G}(y)+n-4\right)\right]^{\alpha}=2^{\alpha} e^{\alpha+1} \\
& +\sum_{\substack{w_{i} w_{j} \in E(G), w_{j} w_{k} \in E(G)}}\left[2 n-8+d_{G}\left(w_{i}\right)+2 d_{G}\left(w_{j}\right)\right. \\
& \left.+d_{G}\left(w_{k}\right)\right]^{\alpha}+\sum_{\substack{b=x y \in E(G), a \in V(G), a \notin\{x, y\}}}\left[e+n-4+d_{G}(x)\right. \\
& \left.+d_{G}(y)\right]^{\alpha} .
\end{aligned}
$$

Note that $d_{G}(a) \leq \Delta(G)$ and $d_{G}(a) \geq \delta(G)$. The equalities hold if and only if $G$ is a regular graph. After simplification, we get

$$
\begin{aligned}
\chi_{\alpha}\left(G^{++-}\right) \geq 2^{\alpha} e^{\alpha+1}+2^{\alpha}\left[\frac{1}{2} M_{1}(G)-e\right] \\
\cdot[n-4+2 \Delta(G)]^{\alpha} \\
+\left[\left(\begin{array}{c}
e+n \\
2
\end{array}\right)-\left(\begin{array}{l}
n \\
2
\end{array}\right)-\left(\begin{array}{l}
e \\
2
\end{array}\right)-2 e\right] \\
\cdot(e+n-4+2 \Delta(G))^{\alpha} .
\end{aligned}
$$

Similarly, we can compute

$$
\begin{aligned}
\chi_{\alpha}\left(G^{++-}\right) \leq 2^{\alpha} e^{\alpha+1}+2^{\alpha}\left[\frac{1}{2} M_{1}(G)-e\right] \\
\cdot(n-4+2 \delta(G))^{\alpha} \\
+\left[\left(\begin{array}{c}
e+n \\
2
\end{array}\right)-\left(\begin{array}{l}
n \\
2
\end{array}\right)-\left(\begin{array}{l}
e \\
2
\end{array}\right)-2 e\right] \\
\cdot(e+n-4+2 \delta(G))^{\alpha} .
\end{aligned}
$$

The equalities in (23) and (24) obviously hold if and only if $G$ is a regular graphs.
Theorem 9. Let $\alpha<0$. Then $\gamma_{1} \leq \chi_{\alpha}\left(G^{-+-}\right) \leq \gamma_{2}$, where

$$
\begin{aligned}
\gamma_{1}= & 2^{\alpha}[e+n-1-2 \Delta(G)]^{\alpha}\left[\left(\begin{array}{l}
n \\
2
\end{array}\right)-e\right] \\
& +2^{\alpha}[n-4+2 \Delta(G)]^{\alpha}\left[\frac{1}{2} M_{1}(G)-e\right] \\
& +(e+2 n-5)^{\alpha}\left[\left(\begin{array}{c}
e+n \\
2
\end{array}\right)-\left(\begin{array}{l}
n \\
2
\end{array}\right)-\left(\begin{array}{l}
e \\
2
\end{array}\right)-2 e\right] \\
\gamma_{2}= & 2^{\alpha}[e+n-1-2 \delta(G)]^{\alpha}\left[\left(\begin{array}{l}
n \\
2
\end{array}\right)-e\right] \\
& +2^{\alpha}[n-4+2 \delta(G)]^{\alpha}\left[\frac{1}{2} M_{1}(G)-e\right] \\
& +(e+2 n-5)^{\alpha}\left[\left(\begin{array}{c}
e+n \\
2
\end{array}\right)-\left(\begin{array}{l}
n \\
2
\end{array}\right)-\left(\begin{array}{l}
e \\
2
\end{array}\right)-2 e\right]
\end{aligned}
$$

the equalities hold if and only if $G$ is a regular graph.

Proof. Since $\left|V\left(G^{-+-}\right)\right|=n+e$ and $\left|E\left(G^{-+-}\right)\right|=\left(\begin{array}{c}e+n \\ 2\end{array}\right)-\left(\begin{array}{l}e \\ 2\end{array}\right)+$ $(1 / 2) M_{1}(G)-4 e$,

$$
\begin{aligned}
\chi_{\alpha}\left(G^{-+-}\right)= & \sum_{a b \in E\left(G^{-+}\right)}\left(d_{G^{-+}}(a)+d_{G^{-+-}}(b)\right)^{\alpha} \\
= & \sum_{\substack{a b \in E\left(G^{-+-}\right), a, b \in V(G)}}\left(d_{G^{-+}}(a)+d_{G^{-+}}(b)\right)^{\alpha} \\
& +\sum_{\substack{a b \in E\left(G^{-+}\right), a, b \in E(G)}}\left(d_{G^{-+-}}(a)+d_{G^{--}}(b)\right)^{\alpha} \\
& +\sum_{\substack{a b \in E\left(G^{-+-}\right), a \in V(G), b \in E(G)}}\left(d_{G^{-+-}}(a)+d_{G^{-+-}}(b)\right)^{\alpha} .
\end{aligned}
$$

Note that $d_{G^{-+}}(a)=e+n-1-2 d_{G}(a)$ for $a \in V(G)$ and $d_{G^{-+}}(a)=d_{G}\left(w_{i}\right)+d_{G}\left(w_{j}\right)+n-4$ for $a \in E(G)$. Then

$$
\begin{aligned}
& \chi_{\alpha}\left(G^{-+-}\right)=\sum_{\substack{a b \in E\left(G^{+-}\right), a, b \in V(G)}}\left[\left(e+n-1-2 d_{G}(a)\right)\right. \\
& \left.+\left(e+n-1-2 d_{G}(b)\right)\right]^{\alpha} \\
& +\sum_{\substack{w_{i} w_{j} \in E(G), w_{j} w_{k} \in E(G), w_{i} \neq w_{k}}}\left[\left(d_{G}\left(w_{i}\right)+d_{G}\left(w_{j}\right)+n-4\right)\right. \\
& \left.+\left(d_{G}\left(w_{j}\right)+d_{G}\left(w_{k}\right)+n-4\right)\right]^{\alpha}
\end{aligned}
$$




$$
\begin{aligned}
& +\sum_{\substack{a b \in E\left(G^{+-}\right), a \in V(G), b=x y \in E(G)}}\left[\left(e+n-1-2 d_{G}(a)\right)\right. \\
& \left.+\left(d_{G}(x)+d_{G}(y)+n-4\right)\right]^{\alpha}=2^{\alpha} \sum_{\substack{a b \notin E E(G), a, b \in V(G)}}[e+n \\
& \left.-1-d_{G}(a)-d_{G}(b)\right]^{\alpha}+\sum_{\substack{w_{i} w_{j} \in E(G), w_{j} w_{k} \in E(G), w_{i} \neq w_{k}}}[2 n-8 \\
& \left.+d_{G}\left(w_{i}\right)+2 d_{G}\left(w_{j}\right)+d_{G}\left(w_{k}\right)\right]^{\alpha}+\sum_{\substack{b=x y \in E(G), a \notin\{x, y\}, a \in V(G)}}[e
\end{aligned}
$$$$
\left.+2 n-5-2 d_{G}(a)+d_{G}(x)+d_{G}(y)\right]^{\alpha} \text {. }
$$

Note that $d_{G}(a) \leq \Delta(G)$ and $d_{G}(a) \geq \delta(G)$. The equalities hold if and only if $G$ is a regular graph.

After simplification, we get

$$
\begin{aligned}
& \chi_{\alpha}\left(G^{-+-}\right) \\
& \geq 2^{\alpha}[e+n-1-2 \Delta(G)]^{\alpha}\left[\left(\begin{array}{l}
n \\
2
\end{array}\right)-e\right] \\
& \quad+2^{\alpha}[n-4+2 \Delta(G)]^{\alpha}\left[\frac{1}{2} M_{1}(G)-e\right] \\
& \quad+(e+2 n-5)^{\alpha}\left[\left(\begin{array}{c}
e+n \\
2
\end{array}\right)-\left(\begin{array}{l}
n \\
2
\end{array}\right)-\left(\begin{array}{l}
e \\
2
\end{array}\right)-2 e\right] .
\end{aligned}
$$

Similarly, we calculate

$$
\begin{aligned}
\chi_{\alpha}\left(G^{-+-}\right) \leq 2^{\alpha}[e+n-1-2 \delta(G)]^{\alpha} \\
\quad \cdot[n-4+2 \delta(G)]^{\alpha}\left[\left(\begin{array}{l}
n \\
2
\end{array}\right)-e\right] \\
\quad+2^{\alpha}\left[\frac{1}{2} M_{1}(G)-e\right] \\
\quad+\left[\left(\begin{array}{c}
e+n \\
2
\end{array}\right)-\left(\begin{array}{l}
n \\
2
\end{array}\right)-(e+2 n-5)^{\alpha}\left(\begin{array}{l}
e \\
2
\end{array}\right)-2 e\right] .
\end{aligned}
$$

If $G$ is a regular graph then we obtain the equalities in (28) and (29).

In fully analogous manner, we also arrive at the following.

Theorem 10. If $\alpha<0$ then

(1) $\gamma_{1} \leq \chi_{\alpha}\left(G^{+--}\right) \leq \gamma_{2}$, where

$$
\begin{gathered}
\gamma_{1}=2^{\alpha} e^{\alpha+1}+2^{\alpha}\left[\left(\begin{array}{l}
e \\
2
\end{array}\right)-\frac{1}{2} M_{1}(G)+e\right] \\
\cdot[n+e-1-2 \Delta(G)]^{\alpha}
\end{gathered}
$$

$$
\begin{aligned}
+ & {\left[\left(\begin{array}{c}
e+n \\
2
\end{array}\right)-\left(\begin{array}{l}
n \\
2
\end{array}\right)-\left(\begin{array}{l}
e \\
2
\end{array}\right)-2 e\right] } \\
\cdot & {[2 e+n-1-2 \Delta(G)]^{\alpha}, } \\
\gamma_{2}= & 2^{\alpha} e^{\alpha+1}+2^{\alpha}\left[\left(\begin{array}{l}
e \\
2
\end{array}\right)-\frac{1}{2} M_{1}(G)+e\right] \\
\cdot & {[n+e-1-2 \delta(G)]^{\alpha} } \\
+ & {\left[\left(\begin{array}{c}
e+n \\
2
\end{array}\right)-\left(\begin{array}{l}
n \\
2
\end{array}\right)-\left(\begin{array}{l}
e \\
2
\end{array}\right)-2 e\right] } \\
& \cdot[2 e+n-1-2 \delta(G)]^{\alpha} ;
\end{aligned}
$$

(2) $\gamma_{1} \leq \chi_{\alpha}\left(G^{--+}\right) \leq \gamma_{2}$, where

$$
\begin{aligned}
\gamma_{1}= & 2^{\alpha}\left[\left(\begin{array}{l}
n \\
2
\end{array}\right)-e\right](n-1)^{\alpha} \\
& +2^{\alpha}\left[\left(\begin{array}{l}
e \\
2
\end{array}\right)-\frac{1}{2} M_{1}(G)+e\right][e-3-2 \Delta(G)]^{\alpha} \\
& +2 e[e+n+2-2 \Delta(G)]^{\alpha} \\
\gamma_{2}= & 2^{\alpha}\left[\left(\begin{array}{l}
n \\
2
\end{array}\right)-e\right](n-1)^{\alpha} \\
& +2^{\alpha}\left[\left(\begin{array}{l}
e \\
2
\end{array}\right)-\frac{1}{2} M_{1}(G)+e\right][e-3-2 \delta(G)]^{\alpha} \\
& +2 e[e+n+2-2 \delta(G)]^{\alpha} ;
\end{aligned}
$$

(3) $\gamma_{1} \leq \chi_{\alpha}\left(G^{-++}\right) \leq \gamma_{2}$, where

$$
\begin{aligned}
\gamma_{1}= & 2^{\alpha}\left[\left(\begin{array}{l}
n \\
2
\end{array}\right)-e\right](n-1)^{\alpha} \\
& +4^{\alpha} \Delta^{\alpha}(G)\left[\frac{1}{2} M_{1}(G)-e\right] \\
& +2 e[n-1+2 \Delta(G)]^{\alpha}, \\
\gamma_{2}= & 2^{\alpha}\left[\left(\begin{array}{l}
n \\
2
\end{array}\right)-e\right](n-1)^{\alpha} \\
& +4^{\alpha} \delta^{\alpha}(G)\left[\frac{1}{2} M_{1}(G)-e\right] \\
& +2 e[n-1+2 \delta(G)]^{\alpha} ;
\end{aligned}
$$

(4) $\gamma_{1} \leq \chi_{\alpha}\left(G^{+-+}\right) \leq \gamma_{2}$, where

$$
\begin{aligned}
\gamma_{1}= & 2^{2 \alpha} e \Delta^{\alpha}(G) \\
& +\left[\left(\begin{array}{l}
e \\
2
\end{array}\right)-\frac{1}{2} M_{1}(G)+e\right][3-2 \Delta(G)]^{\alpha} \\
& +2 e(e+3)^{\alpha},
\end{aligned}
$$




$$
\begin{aligned}
\gamma_{2}= & 2^{2 \alpha} e \delta^{\alpha}(G) \\
& +\left[\left(\begin{array}{l}
e \\
2
\end{array}\right)-\frac{1}{2} M_{1}(G)+e\right][3-2 \delta(G)]^{\alpha} \\
& +2 e(e+3)^{\alpha} .
\end{aligned}
$$

In all the above cases, the equalities hold if and only if $G$ is a regular graph, respectively.

\section{Conclusion}

In this paper, we obtain the sharp lower and upper bounds for general sum-connectivity indices of the semitotal-point graph, the semitotal-line graph, the total graph, and the eight distinct transformation graphs $G^{u v w}$, where $u, v, w \in\{+,-\}$ in terms of the order, minimum degree, and maximum degree of a graph. Moreover, the extremal graphs achieving these bounds have been described.

\section{Conflicts of Interest}

The authors declare that there are no conflicts of interest regarding the publication of this paper.

\section{Acknowledgments}

This research is supported by the Fundamental Research Funds for the Central Universities (nos. 2652015193 and 2652017146) and NSFC of China (no. 11701530).

\section{References}

[1] S. Ji and S. Wang, "On the sharp lower bounds of Zagreb indices of graphs with given number of cut vertices," Journal of Mathematical Analysis and Applications, vol. 458, no. 1, pp. 21-29, 2018.

[2] I. Gutman and N. Trinajstić, "Graph theory and molecular orbitals. Total $\varphi$-electron energy of alternant hydrocarbons," Chemical Physics Letters, vol. 17, no. 4, pp. 535-538, 1972.

[3] M. Randić, "On characterization of molecular branching," Journal of the American Chemical Society, vol. 97, no. 23, pp. 6609-6615, 1975.

[4] B. Bollobás and P. Erdős, "Graphs of extremal weights," Ars Combinatoria, vol. 50, pp. 225-233, 1998.

[5] B. Zhou and N. Trinajstić, "On a novel connectivity index," Journal of Mathematical Chemistry, vol. 46, no. 4, pp. 1252-1270, 2009.

[6] B. Zhou and N. Trinajstić, "On general sum-connectivity index," Journal of Mathematical Chemistry, vol. 47, no. 1, pp. 210-218, 2010.

[7] B. Lučić, N. Trinajstić, and B. Zhou, "Comparison between the sum-connectivity index and product-connectivity index for benzenoid hydrocarbons," Chemical Physics Letters, vol. 475, no. 1-3, pp. 146-148, 2009.

[8] M. Behzad, "A criterion for the planarity of the total graph of a graph," Mathematical Proceedings of the Cambridge Philosophical Society, vol. 63, no. 3, pp. 679-681, 1967.
[9] E. Sampathkumar and S. B. Chikkodimath, "The semi-total graphs of a graph-I," Journal of the Karnatak University, vol. 18, pp. 274-280, 1973.

[10] B. Basavanagoud and P. V. Patil, "A criterion for (non-)planarity of the transformation graph Gxyz when $x y z=-++$," Journal of Discrete Mathematical Sciences \& Cryptography, vol. 13, no. 6, pp. 601-610, 2010.

[11] S. M. Hosamani and I. Gutman, "Zagreb indices of transformation graphs and total transformation graphs," Applied Mathematics and Computation, vol. 247, pp. 1156-1160, 2014.

[12] B. Wu and J. Meng, "Basic properties of total transformation graphs," Journal of Mathematical Study, vol. 34, no. 2, pp. 109116, 2001.

[13] L. Xu and B. Wu, “Transformation graph $G^{-+-, " ~ D i s c r e t e ~ M a t h e-~}$ matics, vol. 308, no. 22, pp. 5144-5148, 2008.

[14] L. Yi and B. Wu, "The transformation graph $G^{++-}$," The Australasian Journal of Combinatorics, vol. 44, pp. 37-42, 2009. 


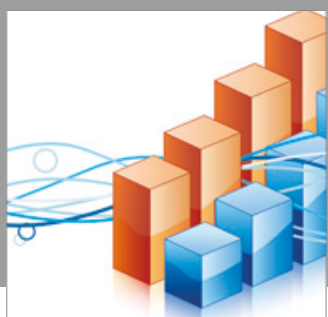

Advances in

Operations Research

vatersals

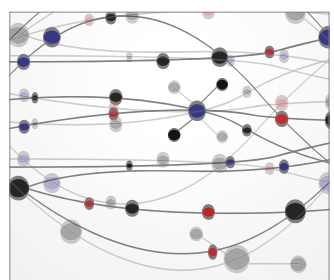

\section{The Scientific} World Journal
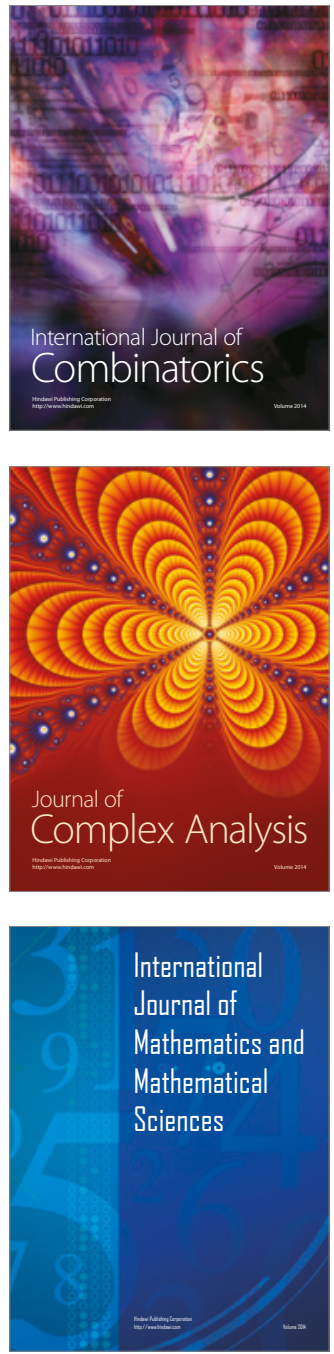
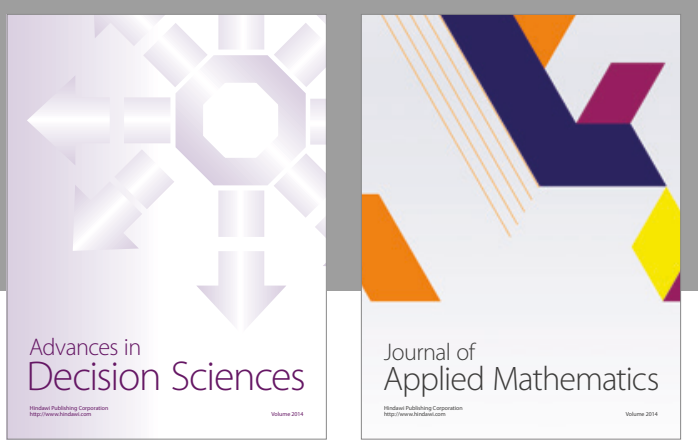

Algebra

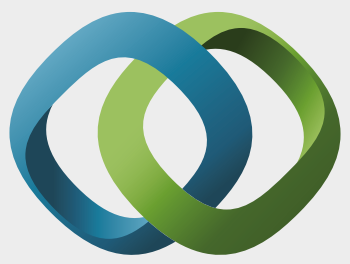

\section{Hindawi}

Submit your manuscripts at

https://www.hindawi.com
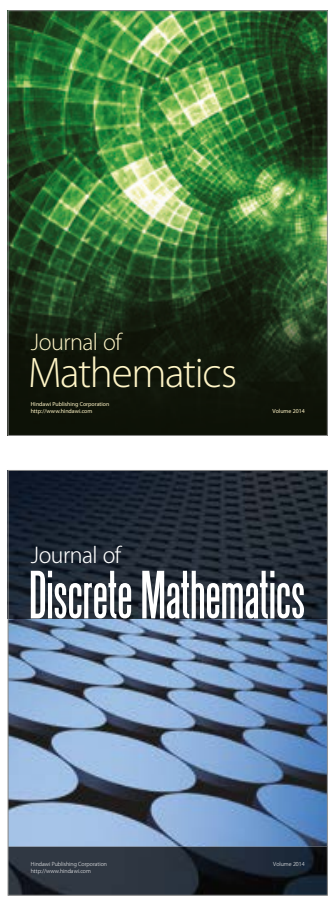

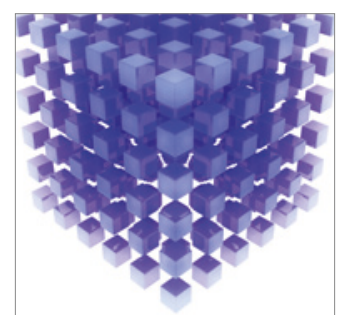

Mathematical Problems in Engineering
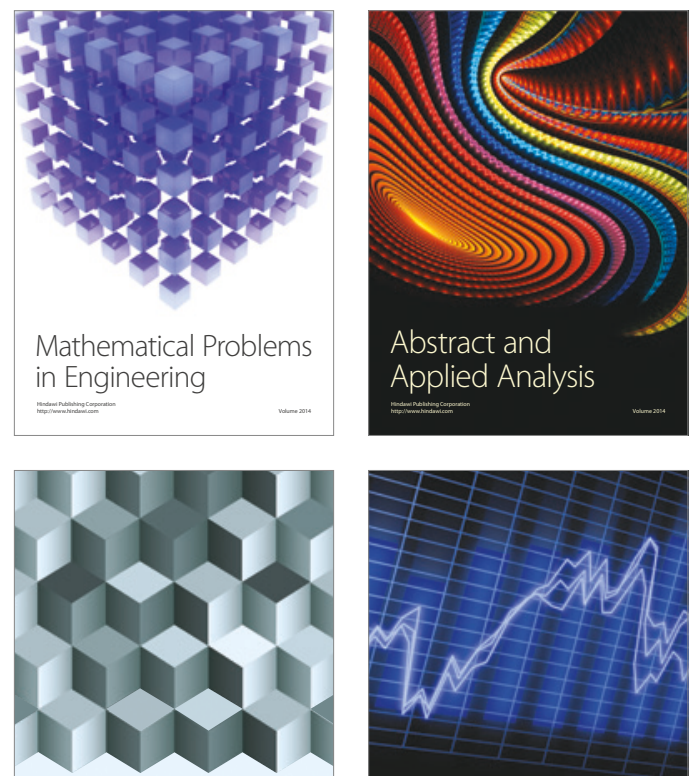

Journal of

Function Spaces

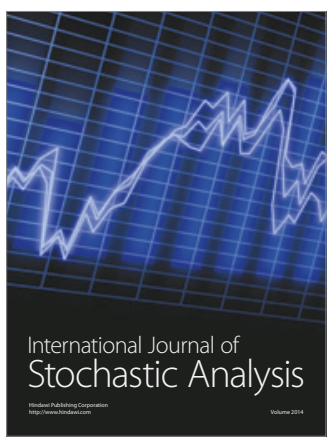

Probability and Statistics
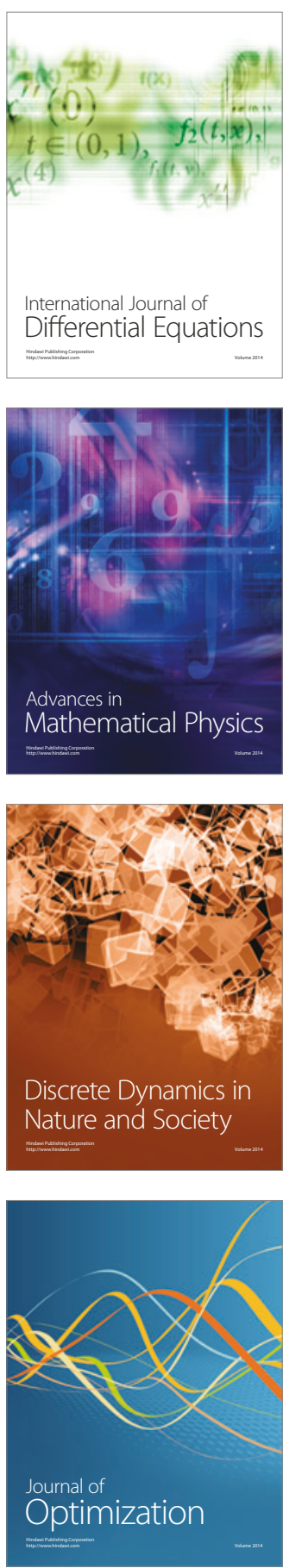